\title{
Complete Sequence Analysis of a Gene (OS-9) Ubiquitously Expressed in Human Tissues and Amplified in Sarcomas
}

\author{
Yan A. Su, Carolyn M. Hutter, Jeffrey M. Trent, and Paul S. Meltzer ${ }^{1}$ \\ Laboratory of Cancer Genetics, National Center for Human Genome Research, National Institutes of Health, \\ Bethesda, Maryland
}

Amplification and overexpression of genes involved in cellular growth control occur frequently in human tumors. Using a chromosome microdissection-based hybrid-selection strategy, we recently identified two novel genes (OS-9 and OS-4) within 12q13-15, a region frequently amplified in human cancers. We now report further characterization of the full-length OS-9 cDNA sequence. This CDNA sequence consists of 2785 bp from which an open reading frame (ORF) with 667 amino-acid residues was deduced. The predicted polypeptide was water soluble and acidic. We also demonstrate that the OS-9 gene encoded a 2.8-kb mRNA transcribed in all 16 human tissues examined, suggesting that OS-9 is ubiquitously expressed in human tissues. OS-9 was coamplified with CDK4 in three of five sarcoma tissues. Homology analysis of the amino-acid sequence reveals significant similarities between OS- 9 and two ORFs deduced from genomic sequences in Caenorhabditis elegans and Saccharomyces cerevisiae. The region of similarity extended over 200 residues (approximately one-third of each ORF), and eight cysteines were conserved in all three ORFs. These observations suggest that this region comprises a functional domain present in a novel evolutionarily conserved gene family defined by OS-9. (1) 1996 Wiley-Liss, Inc.*

Key words: Neoplasm, genetics, chromosome $12 q$

\section{INTRODUCTION}

In human cancers, gene amplification is a common mechanism by which increased dosage of a gene leads to its overexpression. Amplification of cellular oncogenes has been observed in tumor cell lines and primary tumor tissues, suggesting that overexpression of these genes provides tumor cells with a selective growth advantage in vitro and in vivo.

The human osteosarcoma cell line OsA-Cl [1] contains a homogeneously staining region (hsr) composed of sequences derived from 12q13-15. Several amplified genes have been mapped within this hsr, including those for a zinc-finger protein $(G L I)$ [1], a member of the transmembrane 4 superfamily (SAS) $[2,3]$, cyclin-dependent kinase 4 (CDK4) [4], a transcription factor (CHOP) [5], and a modulator of p53 (MDM2) $[6,7]$. Two additional novel genes (OS-9 and $O S-4)$ were recently identified from this hsr [8]. Because the OS-9 gene was shown to be amplified in additional sarcoma specimens, we further characterized its cDNA. The 2785-bp full-length cDNA encodes an open reading frame (ORF) of 667 amino-acid residues. Northern blot analysis demonstrated OS-9 expression in all human tissues examined. Analysis of the amino-acid sequence revealed a significant similarity with two ORFs deduced from genomic sequences of Caenorhabditis elegans [9] and Saccharomyces cerevisiae $[10,11]$.

\section{MATERIALS AND METHODS}

Library Screening and Generation of Plasmid DNA

The generation of the cDNA library (in bacteriophage $\lambda$ ZAPII) highly enriched for transcribed sequences from the $12 \mathrm{q}$ hsr has been previously described [8]. The library was plated at a density of 628 plaque-forming units per $150-\mathrm{mm}$ petri dish in Escherichia coli XL1-blue cells. Plaque lifts and hybridizations were performed by standard methods [12]. A total of 30 plaques was picked after hybridization with the OS-9 probe and used to excise pBluescript (Stratagene, La Jolla, CA) plasmids containing OS-9 cDNA inserts according to the manufacturer's protocol.

\section{5 ' CDNA Synthesis and Cloning}

Synthesis and amplification of the $5^{\prime}$ end of the OS-9 gene were performed according to published methods $[13,14]$ with the following modifications. OsA-Cl was cultured as previously described [8]. Total RNA was isolated by standard methods [15]. Poly $(\mathrm{A})^{+}$RNA was isolated from the total RNA with

\footnotetext{
${ }^{1}$ Corresponding author: NCHGR/NIH, Bldg. 49, Room 4A10, 49 Convent Drive, Bethesda, MD 20892-4470.

Abbreviations: ORF, open reading frame; hsr, homogeneously staining region; CDK4, cyclin-dependent kinase 4; INT-1, integration site1 ; utr, untranslated region; EST, expressed tagged sequence.
} 
Dynabeads Oligo (dT) ${ }_{25}$ (Dynal, Great Neck, New York) following the manufacturer's instructions. The $5^{\prime}$ end of the OS-9 cDNA was synthesized with avian myeloma virus reverse transcriptase (CLONTECH, Palo Alto, CA). Poly(G) was added to the $5^{\prime}$ end of the CDNA with terminal DNA transferase (GIBCO BRL, Gaithersburg, MD). Both OS-9-specific and 5'end primers for the polymerase chain reaction amplification of the CDNA contain the sequence (CUA) 4 at their $5^{\prime}$ ends, so the polymerase chain reaction products were directly cloned into the UDG cloning vector PAMP10 (GIBCO BRL).

\section{DNA Sequencing and Sequence Analysis}

The plasmids containing OS-9 cDNA inserts were sequenced by automated fluorescence sequencing. The sequence analysis of eight plasmid inserts derived from our cDNA library generated a consensus sequence of approximately $2 \mathrm{~kb}$. Using the known sequence, we synthesized and cloned the $5^{\prime}$ end of the OS-9 CDNA. Five plasmids containing 5 '-end cDNA inserts were sequenced and analyzed. Both strands of all of the above-mentioned plasmids were sequenced. Thus, the nucleotide sequence of the fulllength OS- $9 \mathrm{cDNA}$ was determined from the consensus sequence of both strands of 13 plasmids containing overlapping cDNA inserts. Multiple sequence alignments and consensus determinations were performed with DNASTAR software (version 1.58; DNASTAR Inc., Madison, WI). The current sequence databases were searched by using the BLASTN, BLASTP, and FASTA programs $[16,17]$.

\section{Northern and Southern Hybridization}

The OS-9, human $\beta$-actin, CDK4, and integration site-1 (INT-1) probes were described previously $[8,18]$ and labeled by random priming [19]. Northern and Southern hybridizations were performed by standard methods [20]. Filters were washed to a stringency of $0.1 \times$ stanclard saline citrate at $42^{\circ} \mathrm{C}$. The relative intensity of the bands in radiograph was measured by densitometry and analyzed by using IP Lab Spectrum software (Signal Analytics Co., Vienna, VA).

\section{RESULTS}

\section{OS-9 CDNA, Sequence}

Figure 1 shows the full-length $O S-9 \mathrm{cDNA}$ sequence and the predicted sequence of the longest ORF. The 2785-bp nucleotide sequence of the cDNA was determined from the consensus sequence of both strands of a total of 13 plasmids containing overlapping cDNA inserts. The full-length cDNA consisted of three segments: an 85-bp 5' untranslated region (UTR), nt 1-85; a 2001-bp coding region, nt 86-2086; and a 698-bp 3' UTR, nt 2087-2785. This nucleotide sequence encoded an ORF of 667 amino-acid residues. The deduced polypeptide consisted of three parts: an N-terminal hydrophobic sequence (residues
1-31), a cysteine-containing hydrophilic sequence (residues 32-337), and an acidic region with multiple predicted hydrophilic helices (residues 338667). Eight cysteine residues and a possible nuclear targeting signal $[21,22]$ within the ORF are also indicated in Figure 1. The calculated molecular weight of the polypeptide was $75556 \mathrm{Da}$, and the estimated isoelectric point was 4.60 . The cDNA contains a 48 bp nucleotide sequence with $16 \mathrm{GAD}$ trinucleotide repeats (where $D=G, A$, or $T$ ), which encode an acidic domain composed of glutamic acid (E) and aspartic acid (D) (residues 414-429). The 3' UTR was 698 bp long with a polyadenylation signal $13 \mathrm{bp}$ upstream from the polyadenylated site.

\section{Homology Analysis}

Comparison of the OS-9 CDNA sequence with the GenBank database (version 93.0) using the BLASTN program $[16,17]$ revealed identical matches with 15 partial cDNA sequences (expressed, (ESTs)) derived from human fetal brain, spleen, liver, sequence tags and adult liver, ovary, placenta, and a colorectal cancer. The GenBank accession numbers of these ESTs are listed in Figure 1. No significant identity with any known gene was revealed by using the current GenBank and European Molecular Biology Laboratory databases, except for the partial OS-9 sequence, which we previously submitted. However, comparison of the OS-9 ORF with these databases by using the BLASTP program revealed a significant similarity with an ORF (F48E8.4) deduced from cosmid CELF48E8 mapped to chromosome III of C. elegans [9] and a somewhat weaker similarity with an ORF (YD9609.11) predicted from cosmid 9609 on chromosome IV of S. cerevisiae $[10,11]$. BLASTP aligned a 211 amino-acid segment (residues 62-267 (in brackets in Figure 1)) of OS-9 with F48E8.4 $\left(P=1.8 \times 10^{-15}\right)$ and YD9609.11 $(P=$ $\left.1.4 \times 10^{-5}\right)$. Within this homologous region (Figure 2), the identities between OS-9 and F48E8.4, OS-9 and YD9609.11, and F48E8.4 and YD9609.11 were $27.0 \%(57 / 211), 19.6 \%(45 / 230)$, and $22.5 \%(51 / 227)$, respectively, with identity plus similarity being $68.7 \%$ $(145 / 211)$, 53.0\% (122/230), and 60.0\% (137/227), respectively. The most notable feature of this alignment is the conservation of eight cysteine residues in all three ORFs. In addition, the homologous region of all three proteins was preceded by a hydrophobic leader peptide. Furthermore, all three ORFs were predicted to be acidic proteins $(\mathrm{pl}=4.31,4.60$, and 5.12 for YD9609.11, OS-9, and F48E8.4, respectively).

\section{Northern Hybridization}

To evaluate the expression status of OS-9, membranes containing poly $(\mathrm{A})^{+} \mathrm{RNA}$ from multiple adult tissues (CLONTECH) were hybridized with the OS-9 probe and a control probe (human $\beta$-actin) that was used to normalize RNA loading. The result demonstrated that OS-9 was transcribed in all 16 human tissues (Figure 3 ). It was also noted by densitometry 


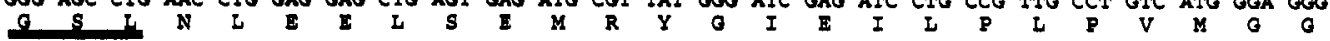

CAO CAM TAC CAC ATO GAA GAT TCA GAO ATC AAM GGT OAA OTC CTC TAT CTC GOC TAC TAC CAA TCA GCC TTC GAC

GTG CAG GTC AAA GTC ATT CGA AGC CCT GCG GAT TTG ATT CGA TTC ATA GAG GAG CTG AAA GGT GGA ACA AAA AAG

TCA CCC CAA CCT ACA GAg GAg GAT CCT GAG CAC AGA GTC CGg GTC CGg GTC ACC AAg CTC CGT CTC GGA GGC CCT

TCCTGGACTGGCTTGCCTCCTCCCCACCTCCCCACCCTGGAACCCCTGAGGGCCAAACAGCAGAGTGGAGCTGAGCTGTGGACCTCTCGGGCAACTCTGT GOGTGTGCGGGCCCTGGGTGATGCTGCTGCCCCTGCTGGCAGCCACCTTGAGACCTCACCGGGCCTGTGATATTTGCTCTCCTGAACTCTCACTCAATC GOGTGTGGGGCCCTGGGTGATGCTGCTGCCCCTGCTGGCAGCCACCTTGAGACCTCACCGGGCCTGTGATATTTGCTCTCCTGAACTCTCACTCAATC CTCTTCCTCTCCTCTGTGGCTTTTCCTGTTATTGTCCCCTAATGATAGGATATTCCCTGCTGCCTACCTGGAGATTCAGTAGGATCTITTGAGTGGAGGT GGGTAGAGAGAGCAAGGAGGGCAGGACACTTAGCAGGCACTGAGCAAGCAGGCCCCCACCTGCCCTTAGTGATGTTTGGAGTCGTTTTACCCTCTTCTAT TGAATTGCCTTGGGATTTCCTTCTCCCLTTCCTGCCCACCCTGTCCCCTACAATTTGTGCTTCTGAGTTGAGGAGCCTTCACCTCTGTTGCTGAGGAAA TGGTAGAATGC

Figure 1. The 2785-bp nucleotide sequence of the OS-9 CDNA with the deduced longest ORF, 667 amino-acid residues (GenBank accession number U41635). The amino-acid sequence is indicated under the nucleotide sequence. A hydrophobic "signal" sequence at the $\mathbf{N}$ terminus of the ORF is underlined. The polypeptide segment ( 211 residues) in brackets is homologous to an ORF (F48E8.4) from C. elegans [9] and an ORF (YD9609.11) from $S$. cerevisiae $[10,11]$. Eight cysteine $(C)$ residues conserved in these ORFs are shown in bold. The $48 \mathrm{nt}$ of trinucleotide repeat GADs
$(D=G, A$, or $T)$ encoding an acidic domain is underlined. The possible nuclear targeting signal is in the shaded box. The asterisk indicates the stop codon. The polyadenylation signal AAUAAA is boxed. " $n$ " represents 49 A residues sequenced. Partial nucleotide sequences within the OS-9 CDNA match perfectly with 15 ESTs derived from the Human Genome Project. The GenBank accession numbers of these ESTs are T52905, T86580, T48324, T57573, T52904, T07270, T54460, T57526, T86757, M85690, T69075, T72427, T48323, R31165, and T24754. 
YD9609.11

F48E8.4

OS -9

Consensus

(61) IVN-MGDDL-ECFIQNASTQLNDVLEDSNEHSNSEKTALLTKTLNQGVK--TIFDKLNERCIFYQAG-FWIYEYCPG

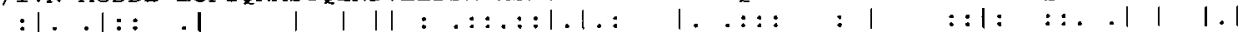
(78) FVTSKGGQKFAC----S--LPDVEDVKKDKPKSSKNP-- -KIYGDALA-ASF--YVDKCVKLRGNHWWSYILCRG

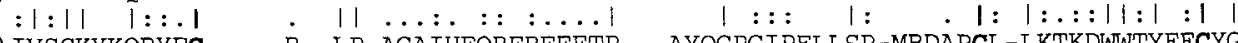
(62) IVSSKYKQRYEC----R--LP-AGAIHFQREREEETP--AYQGPGIPELLSP-MRDAPCL-LKTKDWWTYEFCYG

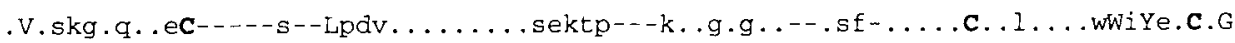

YD9609.11

$\mathrm{F} 48 \mathrm{E} 8.4$

OS-9

Consensus
IEFVOFHGRVNTKTGEIVNRDESLVYRLGKPKANVE--EREFELLYDDVGY-YISEI IGSGDICDVTG--AERMVEIQYV

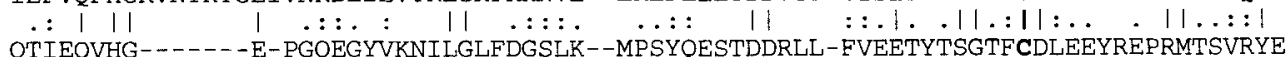
QTIEQVHG--1-DE-PGQEGYVKNILGLFDGSLK-MPSYQESTDDRLL-FVEETYTSGTFCDLEEYREPRMTSVRYE RHIQQYHM-----E-DSEIKGEVLYLGYYQSAFDWDDETAKASKQHRLKRYHSQTYGNGSKCDLNG--RPREAEVRFL ..i.Q.Hg-.---E-......v..LG......-.......ddrl.-y.setYgsG. .CDl.g--.pRm.evry .
YD9609.11

F48E8.4

OS-9

Consensus
CGGSNSGPST-IQWVRETKICVYEAQVTIPELCNL-ELLAKNEDQKNASPILCRMP--AKSKI -GSNSIDLITKY (277)

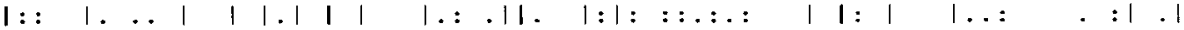
CDAQLSTNEVYISSVVEVKPCQYLMIVKVGTLCRYPEFLPASQSNTKK - IGCQ-PFLRKEDVRQLLERQLEEKQ (281)

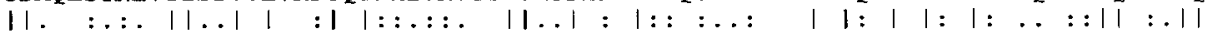
CDEGAGISGDYIDRVDEPLSCSYVLTIRTPRLCPHPLLRPPPSAAPQA--ILCH-PSLQPEEYMAYYVQRQADSKQ (267)
Figure 2. Amino-acid sequence alignment of OS-9 with an ORF (F48E8.4) from C. elegans [9] and an ORF (YD9609.11) from $S$. cerevisiae $[10,11]$. The dashes between the residues indicate gaps to optirnize the alignments. The vertical lines indicate identical residues. The double and single dots between the sequences represent high and low similarities of the residues, respectively. In the consensus sequence, the capital letters indicate identical residues in all three proteins, lowercase letters represent residues conserved in two of the three proteins, and the dots indicate residues different in ail three proteins. The eight conserved cysteine residues are indicated in bold. The numbers in parentheses indicate the positions of the aminoacid residues in the corresponding proteins. The protein alignment was created with the Lipman-Pearson program in the DNASTAR software, Version 1.58.
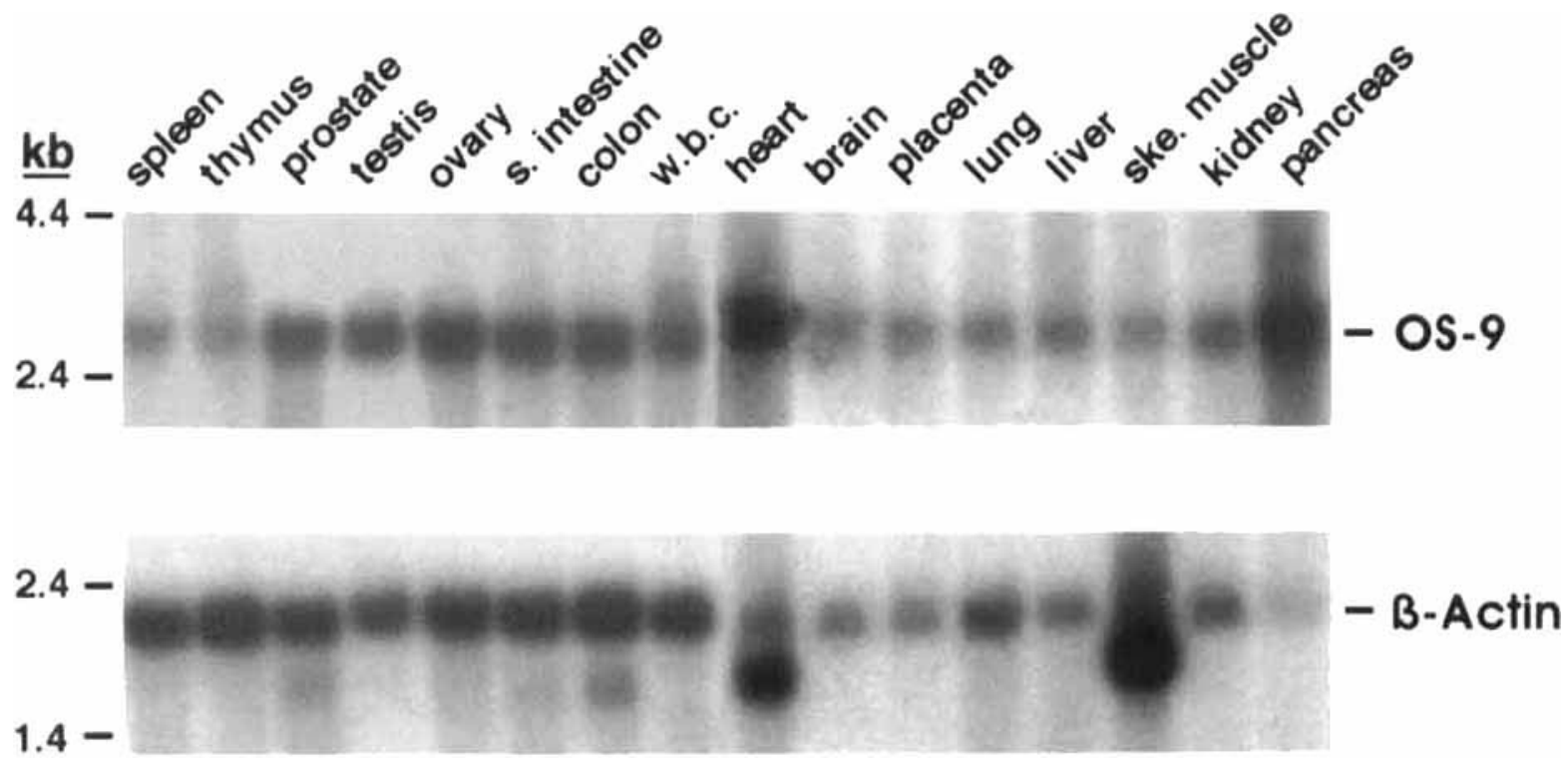

Figure 3. Northern analysis of the OS-9 gene. The OS-9 probe detected $2.8-\mathrm{kb}$ transcripts in all 16 human tissues. The numbers indicate molecular markers $(\mathrm{kb})$. The levels of OS- 9 mRNA were twofold to threefold higher in heart and pancreas and twofold lower in spleen and thymus than in the rest of the tissues. The human $\beta$-actin probe was used as a control for loading error. The weak and strong bands below $\beta$-actin in the lanes of prostate, colon, heart, and skeletal muscle are isoforms of $\beta$ actin mRNA. s, small; w.b.c., white blood cells; ske, skeletal. 
that similar levels of OS-9 mRNA were detected in most tissues except that the levels were twofold to threefold higher in heart and pancreas and twofold lower in spleen and thymus.

\section{Southern Blot Analysis}

To evaluate the OS-9 amplification relative to the closely linked gene $C D K 4$, which falls within $40 \mathrm{~kb}$ of OS-9 [23], Southern blot analysis was performed for DNA samples from six different human sarcoma tissues including five previously noted to carry amplification of the CDK4 gene [18]. The blot was hybridized sequentially with probes for $O S-9, C D K 4$, and finally INT-1 (a gene that maps proximal to the amplified sequence) as a control for loading error. The results (Figure 4) demonstrate that OS-9 was amplified in three of five sarcoma tissues with CDK4 amplification. The relative intensity of each band was measured by densitometry. After normalization relative to $I N T-1$, in comparison to placental DNA, CDK4 was amplified more than fivefold in MFH7, LIP2, and MFH20 and more than threefold in MFH6 and MFH21. OS-9 was amplified to the same degree as CDK4 in MFH20, LIP2, and MFH21 but was not amplified in MFH6 or MFH7. Neither probe detected amplification in MFH9.

\section{DISCUSSION}

We previously identified the OS-9 gene in the human osteosarcoma cell line OsA-Cl and demonstrated that it was amplified and overexpressed relative to human control tissues [8]. We now characterized the OS- 9 CDNA to begin assessing the impact of OS-9 overexpression on tumor phenotype.

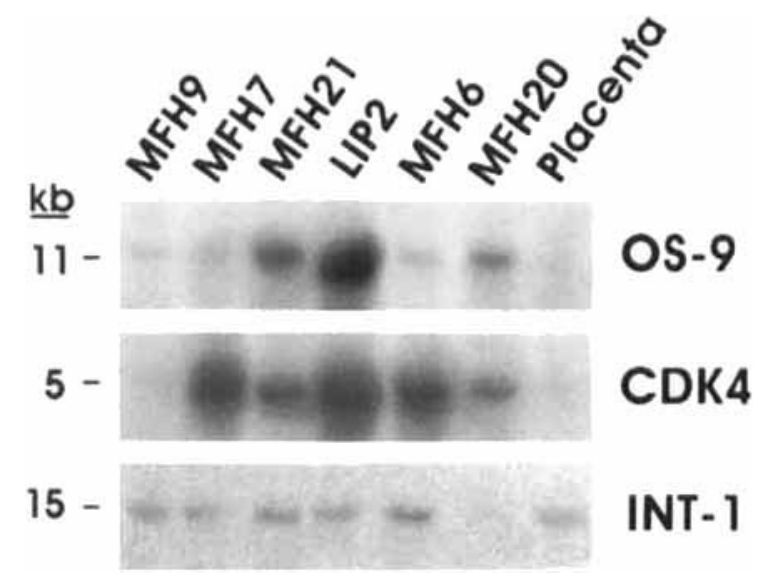

Figure 4. Southern detection of $O S-9$ and $C D K 4$ amplification in human malignant fibrous histocytoma (MFH) and liposarcoma (LIP). OS-9 and CDK4 were coamplified in three of five tissues (MFH21, LIP2, and MFH2O) with CDK4 amplification. Human placenta and a sarcoma without 12q 13-15 amplification (MFH 9) are shown for comparison. The clinical characteristics of these tumor tissues have been described previously [18]. Ten micrograms of the indicated DNA was digested with EcoRI, size-fractionated on an agarose gel, and transferred to a nylon membrane for hybridization. The INT-1 probe was used as a control for loading error.
The conclusion that the 2785-bp nucleotide sequence represents the full-length $O S-9$ cDNA was supported by Northern analysis, which detected a message of approximately $2.8 \mathrm{~kb}$ in all 16 human tissues examined and in OsA-Cl [8]. Additionally, multiple plasmids containing the inserts from $5^{\prime}$ cDNA synthesis had a common $5^{\prime}$ end. The OS-9 ORF predicted a polypeptide with 667 amino-acid residues and a calculated pI of 4.60. This polypeptide contained a short hydrophobic sequence followed by a long cysteine-containing hydrophilic sequence and then multiple predicted hydrophilic helices. Taken together, these data suggest that the OS-9 polypeptide is a water-soluble acidic protein. The hydrophobic sequence ( 31 amino-acid residues) at the $\mathrm{N}$ terminus may act as a signal peptide for membrane insertion. However, the significance of this potential signal sequence as well as the possible nuclear targeting signal remains indeterminate.

The OS-9 gene appears to encode a novel protein belonging to a previously uncharacterized evolutionarily conserved gene family in C. elegans and $S$. cerevisiae. Within this gene family, the similarity between human and $C$. elegans genes $(68.7 \%)$ was greater than that between human and $S$. cerevisiae genes $(53 \%)$, which apparently reflects the greater evolutionary distance between yeast and humans. Nevertheless, the exact alignment of the eight cysteine residues within the conserved regions in all three ORFs strongly suggests that these regions could form similar secondary and tertiary structures that may be essential to the function of the mature proteins. In addition, OS- 9 transcripts were detected in a variety of human normal tissues, suggesting that OS-9 probably has an essential cellular function. The exact alignment of the OS-9 cDNA sequence with the 15 ESTs derived from human fetal and adult tissues further verifies its ubiquitous expression.

We previously demonstrated $O S-9$ co-amplification with CDK4 in OsA-Cl [8]. We now demonstrated the amplification of OS-9 in three of five human sarcoma tissues with $C D K 4$ amplification. Because the CDK4 protein plays a key role in regulating the transition from the $G_{1}$ to $S$ phases of the cell cycle by phosphorylating the retinoblastoma protein, $\mathrm{CDK} 4$ is a likely primary target driving selection of amplificationbearing clones. The closely linked gene OS-9 [23] may be amplified simply by a bystander effect. Alternatively, the co-amplification of $O S-9$ and $C D K 4$ in four of six sarcomas suggests that $O S-9$ has an oncogenic role in a subset of sarcomas. In either case, when amplification of a gene-dense region such as $12 q 13$ 15 occurs, it is important to consider the potential phenotypic effect of each gene, as many are frequently contained in the amplicon. The information presented here should facilitate the functional study of OS- 9 that will be required to evaluate the potential role of this gene in tumorigenesis. It is also noteworthy that the presence of homologous genes 
in C. elegans and S. cerevisiae opens the possibility for functional studies of this novel gene family in these model organisms.

\section{ACKNOWLEDGMENTS}

The authors thank Dr. John Wooton for assistance in the computer analysis of the sequence. We also acknowledge Darryl Leja's help with graphic illustration.

Received October 9, 1995; revised December 5, 1995; accepted December 8, 1995.

\section{REFERENCES}

1. Roberts WM, Douglass EC, Peiper SC, Houghton PJ, Look AT. Amplification of the gli gene in childhood sarcomas. Cancer Res 49:5407--5413, 1989.

2. Meitzer PS, Jankowski SA, Dal cin P, Sandberg AA, Paz IB, Coccia $M A$. Identification and cloning of a novel amplified DNA sequence in human malignant fibrous histocytoma derived from a region of chromosome 12 frequently rearranged in soft tissue tumors. Cell Growth Differ 2:495-501, 1991.

3. Jankowski SA, Mitchell DS, Smith SH, Trent JM, Meitzer PS. SAS, a gene amplified in human sarcomas, encodes a new member of the transmembrane 4 superfamily of proteins. Oncogerie 9:1205-1211, 1994

4. Khatib $2 A$, Matsushime $H$, Valentine $M$, Shapiro DN, Sherr CJ, Look AT. Coamplification of the CDK4 gene with MDM2 and GLI in human sarcomas. Cancer Res 53:5535-5541, 1993.

5. Aman $P$, Ron D, Mandahl N, et al. Rearrangement of the transcription factor gene CHOP in myxoid liposarcomas with $\mathrm{t}(12 ; 16)\{q 13 ; p 11)$. Genes Chromosom Cancer 5:278-285. 1992

6. Oliner JD, Kinzler KW, Meltzer PS, George DL, Vogelstein B. Amplification of a gene encoding a p53-associated protein in human sarcomas. Nature 358:80-83, 1992

7. Leach FS, Tokino T, Meltzer PS, et al. p53 Mutation and MDM2 amplification in human soft tissue sarcomas. Cancer Res 53:22312234, 1993.

8. SU YA, Trent JM, Guan XY, Meltzer PS. Direct isolation of genes encoded within a homogeneously staining region by chromo- some microdissection. Proc Natl Acad Sci USA 91:91219125,1994

9. Wilson $R$, Ainscough $R$, Anderson $\mathrm{K}$, et al. $2.2 \mathrm{Mb}$ of contiguous nucleotide sequence from chromosome III of $C$. elegans. Nature 368:32-38, 1994

10. Barrell B, Rajandream MA. GenBank accession number: $Z 49209$, 1995

11. Brandt P. GenBank accession number: X84162, 1995.

12. Sambrook J, Fritsch EF, Maniatis T. Molecular Cloning: A Laboratory Manual, 2nd edition. Cold Spring Harbor Laboratory Press, Cold Spring Harbor, New York, 1989, pp. 2.108-2.111.

13. Frohman MA, Dush MK, Martin GR. Rapid production of fulllength CDNAs from rare transcripts: Amplification using a single gene-specific oligonucleotide primer. Proc Natl Acad Sci USA 85:8998-9002, 1988.

14. Belyavski A, Vinogradova T, Rajewsky K. PCR-based cDNA library construction: General CDNA libraries at the level of a few cells. Nucleic Acids Res 17:2919-2932, 1989.

15. Sambrook J, Fritsch EF, Maniatis T. Molecular Cloning: A Laboratory Manual, 2nd edition. Cold Spring Harbor Laboratory Press, Cold Spring Harbor, New York, 1989, pp. 7.6-7.9.

16. Pearson WR, Lipman DJ. Improved tools for biological sequence comparison. Proc Natl Acad Sci USA 85:2444-2448, 1988.

17. Altschul S, Gish W, Miller W, Myers EW, Lipman DJ. Basic local alignment search tool. J Mol Biol 215:403-410, 1990.

18. Smith SH, Weiss SW, Jankowski SA, Coccia MA, Meltzer PS. SAS amplification in soft tissue sarcomas. Cancer Res 52:37463749, 1992

19. Feinberg $A P$, Vogelstein $B$. A technique for radio-labeling DNA restriction endonuclease fragments to high specific activity. Anal Biochem 132:6-13, 1983.

20. Brown T. Northern hybridization of RNA fractionated by agarose-formaldehyde gel electrophoresis. In: Ausubel FM, Brent R, Kingston RE, et al. (eds), Current Protocols in Molecular Biology, Green Publishing and John Wiley \& Sons, New York, 1993, pp. 4.9.1-4.9.8

21. Kalderon DB, Roberts $L$, Richardson WD, Smith $A E$. A short amino acid sequence able to specify nuclear location. Cell 39:499509, 1984

22. Robbins J, Dilworth SM, Laskey RA, Dingwall C. Two interdependent basic domains in nucleoplasmin nuclear targeting sequence: Identification of a class of bipartite nuclear targeting sequence. Cell 64:615-623, 1991

23. Elkahloun AG, Meltzer PS, Guan XY, McNinch JS, Trent JM, de Jong PJ. Isolation of a cosmid sublibrary for a region of chromosome 12 frequently amplified in human cancers using a complex chromosome microdissection probe. Genomics, in press. 